\title{
Statistical theory of materials with nanoscale phase separation
}

\author{
V.I. Yukalov ${ }^{1 *}$ and E.P. Yukalova ${ }^{2}$ \\ ${ }^{1}$ Bogolubov Laboratory of Theoretical Physics, \\ Joint Institute for Nuclear Research, Dubna 141980, Russia \\ ${ }^{2}$ Laboratory of Information Technologies, \\ Joint Institute for Nuclear Research, Dubna 141980, Russia
}

\begin{abstract}
Materials with nanoscale phase separation are considered. These materials are formed by a mixture of several phases, so that inside one phase there exist nanosize inclusions of other phases, with random shapes and random spatial locations. A general approach is described for treating statistical properties of such materials with nanoscale phase separation. Averaging over the random phase configurations, it is possible to reduce the problem to the set of homogeneous phase replicas, with additional equations defining the geometric weights of different phases in the mixture. The averaging over phase configurations is mathematically realized as the functional integration over the manifold indicator functions. This procedure leads to the definition of an effective renormalized Hamiltonian taking into account the existence of competing phases. Heterophase systems with mesoscopic phase separation can occur for different substances. The approach is illustrated by the model of a high-temperature superconductor with non-superconducting admixture and by the model of a ferroelectric with paraelectric random inclusions.
\end{abstract}

Keywords: Nanoscale phase separation, High-temperature heterogeneous superconductor, Ferroelectric with paraelectric admixture

*Corresponding author: V.I. Yukalov

E-mail: yukalov@theor.jinr.ru

Phone: 7(496)216-3947

\section{Introduction}

Many materials display heterogeneous phase structure, when inside a sample with one thermodynamic phase there occur inclusions of another phase. The known examples of such materials are high-temperature superconductors, where superconducting phase can coexist with nonsuperconducting phase [1-5], or ferroelectrics, where inside ferroelectric phase there can exist the germs of paraelectric phase [6-14]. A number of other examples can be found in the review articles $[15,16]$. Such materials with heterogeneous phase structure are called heterophase. Since the inclusions of the other phases are usually of nanosizes, being in between the microscopic mean interatomic distance and the macroscopic size of the whole sample, they are termed nanoscale or mesoscopic germs. The occurrence of such germs in a sample is called nanoscale or mesoscopic phase separation. 
In the present paper, we consider such heterophase materials. We concentrate on the situation, when the inclusions of the competing phases do not form regular spatial structures but are randomly distributed in space and have random shapes. The germs are of nanosizes and are mesoscopic, which means that the typical size $l_{g}$ of such germs is much larger than the mean interatomic distance $a$, but essentially smaller than the system length $L$, so that $a \ll l_{g} \ll L$. The germs are not necessarily compact, but may have various shapes. The typical size $l_{g}$ should be understood as the average size, since in reality the germs may possess a rather wide distribution of nanoscopic sizes, being in that sense multiscale.

In Sec. 2, we suggest a general approach for treating such materials with nanoscale phase separation. The approach can be applied to different matters. Its application is given in Sec. 3 for heterophase ferroelectrics and in Sec. 4, for heterophase superconductors. The heterophase structure of materials makes their properties much richer than would be the properties of pure-phase systems. Section 5 is a brief conclusion.

\section{General approach}

Let us consider a sample that is spatially separated into two different thermodynamic phases, whose regions are randomly intermixed in the space. The most general way of characterizing any kind of such a separation is by means of the Gibbs equimolecular separating surface $[17,18]$. Then the total volume $V$ of the sample is the sum of the total volumes of the particular phases, and the total number of particles $N$ is also the corresponding sum:

$$
V=V_{1}+V_{2}, \quad N=N_{1}+N_{2} .
$$

The snap-shot spatial locations of the phase regions are described by the manifold indicator function

$$
\xi_{\nu}(\mathbf{r})= \begin{cases}1, & \mathbf{r} \in \mathbb{V}_{\nu} \\ 0, & \mathbf{r} \notin \mathbb{V}_{\nu}\end{cases}
$$

The notation $V_{\nu} \equiv$ mes $\mathbb{V}_{\nu}$ is used. The statistical ensemble of the phase separated system is the pair $\{\mathcal{H}, \hat{\rho}(\xi)\}$, with the fiber space of microstates

$$
\mathcal{H}=\mathcal{H}_{1} \bigotimes \mathcal{H}_{2}
$$

being the tensor product of weighted Hilbert spaces $[15,16]$. The statistical operator $\hat{\rho}(\xi)$ satisfies the normalization condition

$$
\operatorname{Tr} \int \hat{\rho}(\xi) \mathcal{D} \xi=1
$$

where the trace is over the quantum degrees of freedom and the functional integration is over the topological space $\{\xi\}$ of all manifold indicator functions. The integration over the manifold indicator functions implies the averaging over random phase configurations.

To define the form of the statistical operator, we need to deal with a representative statistical ensemble uniquely characterizing the considered statistical system. For this purpose, we recollect the definition of the internal energy

$$
E=\operatorname{Tr} \int \hat{\rho}(\xi) \hat{H}(\xi) \mathcal{D} \xi
$$


that is the average of the energy Hamiltonian $\hat{H}(\xi)$. Similarly, there can exist the values

$$
C_{i}=\operatorname{Tr} \int \hat{\rho}(\xi) \hat{C}_{i}(\xi) \mathcal{D} \xi
$$

that are the averages of some constraint operators $\hat{C}_{i}(\xi)$. As an example of the latter, one can keep in mind the number-of-particle operator associated with the conservation of the average total number of particles.

With the given constraints, we construct the information functional

$$
\begin{gathered}
I[\hat{\rho}(\xi)]=\operatorname{Tr} \int \hat{\rho}(\xi) \ln \hat{\rho}(\xi) \mathcal{D} \xi+\lambda_{0}\left[\operatorname{Tr} \int \hat{\rho}(\xi) \mathcal{D} \xi-1\right]+ \\
+\beta\left[\operatorname{Tr} \int \hat{\rho}(\xi) \hat{H}(\xi) \mathcal{D} \xi-E\right]+\sum_{i} \lambda_{i}\left[\operatorname{Tr} \int \hat{\rho}(\xi) \hat{C}_{i}(\xi) \mathcal{D} \xi-C_{i}\right]
\end{gathered}
$$

in which the first term is the Shannon information and $\lambda_{0}, \beta$, and $\lambda_{i}$ are the Lagrange multipliers guaranteeing the validity of conditions (4) to (6). The statistical operator is obtained from the principle of minimal information, that is, from minimizing the information functional, which yields

$$
\hat{\rho}(\xi)=\frac{1}{Z} \exp \{-\beta H(\xi)\}
$$

with the grand Hamiltonian

$$
H(\xi)=\hat{H}(\xi)-\sum_{i} \mu_{i} \hat{C}_{i}(\xi)
$$

and partition function

$$
Z=\operatorname{Tr} \int \exp \{-\beta H(\xi)\} \mathcal{D} \xi,
$$

where $\beta \equiv 1 / T$ is inverse temperature and $\mu_{i} \equiv-\lambda_{i} T$.

Accomplishing the functional integration over the manifold characteristic functions gives the effective renormalized Hamiltonian $\tilde{H}$ defined by the equality

$$
\exp \{-\beta \widetilde{H}\}=\int \exp \{-\beta H(\xi)\} \mathcal{D} \xi
$$

Then the partition function (10) reduces to

$$
Z=\operatorname{Tr} \exp \{-\beta \widetilde{H}\}
$$

with the trace over the quantum degrees of freedom.

The values

$$
w_{\nu}=\int \xi_{\nu}(\mathbf{r}) \mathcal{D} \xi \quad(\nu=1,2)
$$

enjoying the properties

$$
w_{1}+w_{2}=1, \quad 0 \leq w_{\nu} \leq 1,
$$

are the geometric probabilities of the phases, serving as the minimizers of the grand potential

$$
\Omega=-T \ln \operatorname{Tr} e^{-\beta \widetilde{H}}
$$

under the normalization condition (14).

In this way, for a considered heterophase substance, we need to find the effective Hamiltonian $\tilde{H}$, using relation (11), after which the following calculations deal with the standard methods of statistical physics, as in expression (15) for the grand potential. 


\section{Heterophase ferroelectrics}

As the first illustration of the approach, let us consider ferroelectrics with hydrogen bonds $[10,19]$, such as $\mathrm{HCl}$ and DCl. The Hamiltonian for this material can be obtained starting from the usual Hamiltonian of charged particles in a lattice of double wells $[19,20]$. For hydrogenbonds ferroelectrics the charged particles are protons. The scheme of the derivation is as follows. The field operators of the particles are expanded over Wannier functions as

$$
\psi(\mathbf{r})=\sum_{n j} c_{n j} \varphi_{n j}(\mathbf{r})
$$

where the multi-index $n$ labels energy bands with energies $E_{n}$ and the index $j=1,2, \ldots$ enumerates lattice sites. The material is insulating, with well localized Wannier functions, so that particles do not jump between the lattice sites,

$$
c_{m i}^{\dagger} c_{n j}=\delta_{i j} c_{m j}^{\dagger} c_{n j}
$$

The unity filling factor is assumed, leading to the unipolarity condition

$$
\sum_{n} c_{n j}^{\dagger} c_{n j}=1, \quad c_{m j} c_{n j}=0
$$

Taking into account two lowest bands allows us to invoke the quasispin representation

$$
c_{1 j}^{\dagger} c_{1 j}=\frac{1}{2}+S_{j}^{x}, \quad c_{2 j}^{\dagger} c_{2 j}=\frac{1}{2}-S_{j}^{x}, \quad c_{1 j}^{\dagger} c_{2 j}=S_{j}^{z}-i S_{j}^{y}
$$

with the operators $S_{j}^{\alpha}$ satisfying the spin-half algebra.

Following the general scheme of Sec. 2, we average over phase configurations obtaining the effective Hamiltonian

$$
\widetilde{H}=H_{1} \bigoplus H_{2}
$$

in which the Hamiltonian replicas are

$$
H_{\nu}=w_{\nu} N E_{0}+\frac{w_{\nu}^{2}}{2} \sum_{i \neq j} A_{i j}-w_{\nu} \Omega_{0} \sum_{j} S_{j}^{x}+w_{\nu}^{2} \sum_{i \neq j}\left(B_{i j} S_{i}^{x} S_{j}^{x}-I_{i j} S_{i}^{z} S_{j}^{z}\right) .
$$

Here $E_{0} \equiv\left(E_{1}+E_{2}\right) / 2$, the tunneling frequency $\Omega_{0} \equiv E_{2}-E_{1}$, and $A_{i j}, B_{i j}, I_{i j}$ are the matrix elements of the pair interaction potential of particles $[19,20]$. The phase weights $w_{\nu}$ are defined by minimizing the grand potential (15).

The phases are distinguished by the electric polarization, so that ferroelectric possesses a finite polarization and paralectric, zero polarization. The polarization is proportional to the mean imbalance

$$
s_{\nu} \equiv \frac{2}{N} \sum_{j=1}^{N}\left\langle S_{j}^{z}\right\rangle_{\nu}
$$

which, hence, serves as an order parameter. The ferroelectric phase, with $\nu=1$, is characterized by a nonzero order parameter

$$
s_{1} \neq 0
$$

while the paraelectric phase, with $\nu=2$, by the zero order parameter

$$
s_{2} \equiv 0
$$


We calculated the ferroelectric order parameter (23) in the mean-field approximation, keeping in mind that $\Omega_{0}$ and $B_{i j}$ are small as compared to $I_{i j}$. The behavior of the order parameter (23) essentially depends on the quantity

$$
u \equiv \frac{A}{I} \quad\left(A \equiv \frac{1}{N} \sum_{i \neq j} A_{i j}, \quad I \equiv \frac{1}{N} \sum_{i \neq j} I_{i j}\right)
$$

characterizing the ratio of the repulsive-interaction intensity to that of attractive interactions. Repulsive interactions oppose the system ordering, while the attractive interactions are responsible for this ordering. Therefore quantity (25) plays the role of a disorder parameter.

The behavior of the ferroelectric order parameter (23), as a function of temperature, is shown in Fig. 1. Temperature is measured in units of $I$. For negative values of $u$, phase separation cannot occur, and the sample is pure, with a second-order phase transition at the critical temperature $T_{c}=0.5$. Nanoscale phase separation occurs for $u>0$. When $0<u<$ $3 / 2$, the phase transition to the paraelectric phase is of first order, happening in the range of temperatures

$$
\frac{1}{8} \leq T_{0} \leq \frac{1}{2}
$$

For $u>3 / 2$ the phase transition is of second order, occurring at the tricritical point $T_{c}^{*}=$ $1 / 8$. As is seen, the appearance of the heterophase states makes the phase-transition picture essentially richer that it would be for a pure ferroelectric phase, where there can happen only the second-order phase transition at $T_{c}=0.5$.

\section{Heterophase superconductors}

Mesoscopic phase separation in high-temperature superconductors has been documented in many experiments [1-5,21-23]. Yet before the high-temperature superconductivity was discovered [24], there had been suggestions $[25,26]$ for the possibility of nanoscopic phase separation in superconductors. These and the following theoretical models [25-31] have shown that the arising heterophase states in superconductors are quite natural, rendering the material more stable, as compared to the pure-phase sample.

The origin of high-temperature superconductivity has not yet been unambiguously clarified. There exist several mechanisms attempting to explain this phenomenon. The aim of the present paper is not to compare different models of describing high-temperature superconductivity or to study their relation to experiment, but our goal is to analyze, in the frame of one model, based on one chosen approximation, how the appearance of nanoscale phase separation changes the properties of the system, as compared to the same approximation, but without taking into account the phase separation. For this purpose, we start with the standard Hamiltonian of charged particles, whose field operators are expanded over the basis of Bloch functions

$$
\psi_{s}(\mathbf{r})=\sum_{k} c_{s}(\mathbf{k}) \varphi_{k}(\mathbf{r})
$$

where the index $s$ denotes spin, while $\mathbf{k}$ is quasimomentum. The problem can be simplified by involving the Bardeen-Cooper-Schrieffer restriction

$$
c_{s}^{\dagger}(\mathbf{k}) c_{r}(\mathbf{p})=\delta_{s r} \delta_{k p} c_{s}^{\dagger}(\mathbf{k}) c_{s}(\mathbf{k}), \quad c_{s}(\mathbf{k}) c_{r}(\mathbf{p})=\delta_{-s r} \delta_{-k p} c_{s}(\mathbf{k}) c_{-s}(-\mathbf{k})
$$


Assuming the occurrence of nanoscale phase separation and averaging over phase configurations, according to Sec. 2, we come to the effective Hamiltonian (20), with the Hamiltonian replicas

$$
\begin{gathered}
H_{\nu}=w_{\nu} \sum_{s} \sum_{k p}\left[t_{\nu}(\mathbf{k}, \mathbf{p})-\mu \delta_{k p}\right] c_{s}^{\dagger}(\mathbf{k}) c_{s}(\mathbf{p})+ \\
+\frac{w_{\nu}^{2}}{2} \sum_{s s^{\prime}} \sum_{k k^{\prime}} \sum_{p p^{\prime}} V_{\nu}\left(\mathbf{k}, \mathbf{k}^{\prime}, \mathbf{p}^{\prime}, \mathbf{p}\right) c_{s}^{\dagger}(\mathbf{k}) c_{s^{\prime}}\left(\mathbf{k}^{\prime}\right) c_{s^{\prime}}\left(\mathbf{p}^{\prime}\right) c_{s}(\mathbf{p}),
\end{gathered}
$$

defined on the space $\mathcal{H}_{\nu}$, and in which $t_{\nu}(\mathbf{k}, \mathbf{p})$ is the transport matrix, $\mu$ is chemical potential, and $V_{\nu}\left(\mathbf{k}, \mathbf{k}^{\prime}, \mathbf{p}^{\prime}, \mathbf{p}\right)$ is the interaction vertex.

The phases are distinguished by the order parameters that are given either by the anomalous averages

$$
\sigma_{\nu}(\mathbf{k}) \equiv\left\langle c_{-s}(-\mathbf{k}) c_{s}(\mathbf{k})\right\rangle_{\nu}
$$

or by the related gaps

$$
\Delta_{\nu}(\mathbf{k})=w_{\nu} \sum_{p} J_{\nu}(\mathbf{k}, \mathbf{p}) \sigma_{\nu}(\mathbf{p})
$$

where $\nu=1,2$. Statistical averages for the superconducting phase are defined in the space $\mathcal{H}_{1}$ with broken gauge symmetry, because of which

$$
\sigma_{1}(\mathbf{k}) \not \equiv 0, \quad \Delta_{1}(\mathbf{k}) \not \equiv 0
$$

while for the normal phase, gauge symmetry of microstates in $\mathcal{H}_{2}$ is preserved, yielding

$$
\sigma_{2}(\mathbf{k}) \equiv 0, \quad \Delta_{2}(\mathbf{k}) \equiv 0
$$

Next, we use the Hartree-Fock-Bogolubov approximation, which, together with the BardeenCooper-Schrieffer restriction (27), gives the superconducting gap equation

$$
\Delta_{1}(\mathbf{k})=\frac{w_{1}^{2}}{2} \sum_{p} J_{1}(\mathbf{k}, \mathbf{p}) \frac{\Delta_{1}(\mathbf{p})}{E_{1}(\mathbf{p})} \tanh \frac{w_{1} E_{1}(\mathbf{p})}{2 T}
$$

in which $E_{1}(\mathbf{p})$ is the Bogolubov spectrum of excitations. The similar equation for the normal phase is trivial, giving zero gap, according to condition (32). As is seen, the equation contains the phase probability $w_{1}$, characterizing the geometric weight of the superconducting phase. In order that the heterophase superconductor be stable, the grand potential (15) is to be minimal with respect to this weight. The positivity of the second derivative of the grand potential plays the role of stability condition and requires the existence of effective Coulomb interactions $Q>0$. That is: nanoscale phase separation in superconductors can be thermodynamically stable only in the presence of repulsive Coulomb interactions.

Superconductivity, as such, presupposes that the gap is not identically zero, in agreement with definition (31). This leads to the superconductivity criterion

$$
\frac{|\alpha|^{2}}{\omega_{0}^{2}}-w_{1} Q>0
$$

where $\alpha$ is the charge-lattice coupling and $\omega_{0}$ is the characteristic lattice frequency.

In a pure-phase sample, where $w_{1}=1$, strong Coulomb interactions may hamper the existence of superconductivity, increasing the second term of inequality (34). But the same sample 
can become superconducting, if phase separation occurs, so that $w_{1}$ becomes less than one, thus diminishing the second term in criterion (34). Therefore: nanoscale phase separation enables the appearance of superconductivity even if it were impossible in pure-phase matter.

Introducing the dimensionless parameter $\mu^{*}$, the superconductivity criterion (34) can be written in the form

$$
1-w_{1} \mu^{*}>0 \quad\left(\mu^{*} \equiv \frac{\omega_{0}^{2}}{|\alpha|^{2}} Q\right)
$$

In the case of good conductors, $\mu^{*} \ll 1$, and the criterion is easily satisfied. However, for bad conductors, $\mu^{*}>1$, so that criterion (35) can be broken in a pure sample, where $w_{1}=1$. But, in the presence of phase separation, when $w_{1}<1$, this criterion can again be valid. Hence: nanoscale phase separation facilitates the occurrence of superconductivity in bad conductors.

Keeping in mind cuprates [32-35], let us consider superconductivity in a two-dimensional plane, with a square lattice of lattice spacing $a$. We shall use the dimensionless quasimomenta $\mathbf{k} \equiv \mathbf{p} a$. The gap in high-temperature superconductors is usually anisotropic and can be represented as an expansion

$$
\Delta_{1}(\mathbf{k})=\sum_{i} \Delta_{i} \chi_{i}(\mathbf{k})
$$

over a basis of functions characterizing the existing lattice symmetry. For a square lattice, one has the functions

$$
\begin{array}{ll}
\chi_{1}(\mathbf{k})=1 & (\text { s symmetry }), \\
\chi_{2}(\mathbf{k})=\frac{1}{2}\left(\cos k_{1}+\cos k_{2}\right) & \left(s^{*} \text { symmetry }\right), \\
\chi_{3}(\mathbf{k})=\cos k_{1} \cdot \cos k_{2} & \left(s_{x y} \text { symmetry }\right), \\
\chi_{4}(\mathbf{k})=\sin k_{1} \cdot \sin k_{2} & \left(d_{x y} \text { symmetry }\right), \\
\chi_{5}(\mathbf{k})=\frac{1}{2}\left(\cos k_{1}-\cos k_{2}\right) & \left(d_{x^{2}-y^{2}} \text { symmetry }\right) .
\end{array}
$$

The prevalence of this or that symmetry is prescribed by the largest density of states. Accepting the parameters typical of cuprates, we come to the conclusion that the largest density of states occurs for the mixture of $s$ and $d_{x^{2}-y^{2}}$ symmetries.

Finally, assuming that the superconducting weight is proportional to the doping, we calculate the superconducting transition temperature $T_{c}$, which is presented in Fig. 2 . As we see: nanoscale phase separation results in a bell shape of the superconducting temperature as a function of the superconducting phase weight.

\section{Conclusion}

Nanoscale phase separation can occur in different types of matter. When the phase separation arises in the form of nanoscale germs of competing phases, randomly distributed in space, then it is possible to introduce the averaging over the random phase configurations and reduce the consideration to the study of an effective Hamiltonian renormalized with phase weights that serve as the minimizers of the grand potential. Therefore the phase separated sample can be more stable than the pure-phase one. We illustrated the general approach by two models taking into account the phase separation. The first model is a ferroelectric with the germs 
of paraelectric phase. The second example is a superconductor with the germs of normal phase. The occurrence of the nanoscale phase separation in materials essentially changes their properties, yielding a number of effects that would be absent in pure-phase samples, without phase separation.

The authors declare that they have no conflict of interest.

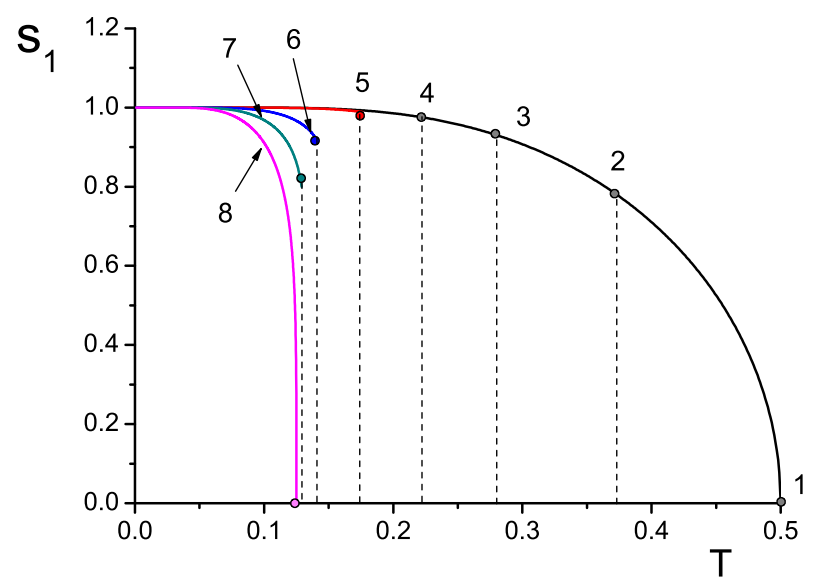

Figure 1: Ferroelectric order parameter as a function of temperature (in units of $I$ ) for different disorder parameters: (1) $u=0$; (2) $u=0.1$; (3) $u=0.3$; (4) $u=0.4$; (5) $u=0.51$; (6) $u=0.75$; (7) $u=1$; (8) $u=1.5$. The points mark the temperatures of ferroelectric-paraelectric phase transitions.

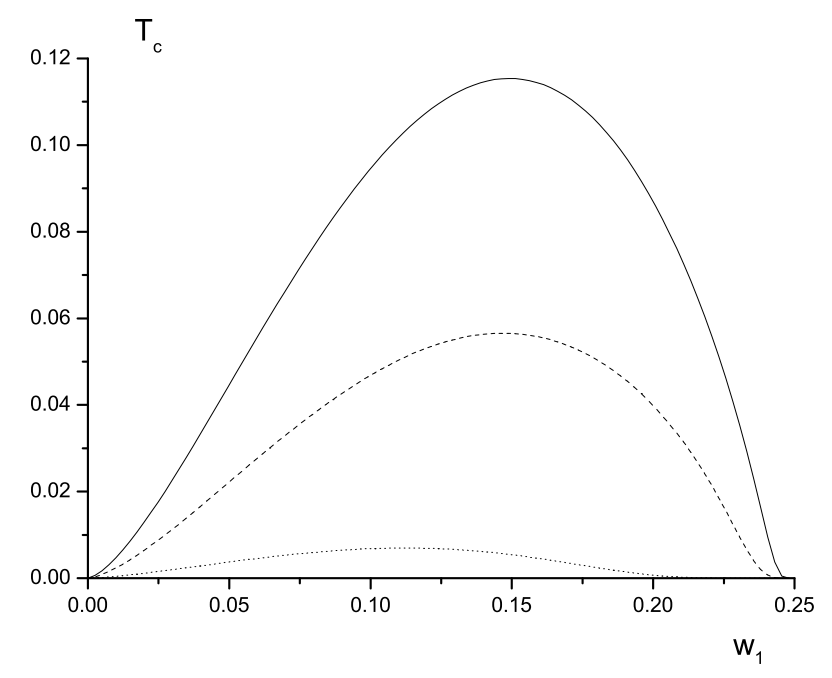

Figure 2: Critical temperature $T_{c}$ (in units of $\omega_{0}$ ) as a function of the superconducting weight $w_{1}$ for different effective couplings: $\lambda=1$ (dotted line); $\lambda=5$ (dashed line); $\lambda=10$ (solid line). 


\section{References}

[1] Phillips, J.C.: Physics of High-Tc Superconductors. Academic, Boston (1989).

[2] Bianconi, A., Saini, N.L., Rossetti, T., Lanzaras, A., Perali, A., Missori, M.: Stripe structure in the $\mathrm{CuO}_{2}$ plane of perovskite superconductors. Phys. Rev. B 54, 12018-12021 (1996).

[3] Bianconi, A., Lusignoli, M., Saini, N.L., Bordet, P., Kvick, A., Radaelli, P.G.: Stripe structure of the $\mathrm{CuO}_{2}$ plane in $\mathrm{Bi}_{2} \mathrm{Sr}_{2} \mathrm{CaCu}_{2} \mathrm{O}_{8+y}$ by anomalous X-ray diffraction. Phys. Rev. B 54, 4310-4314 (1996).

[4] Fratini, M., Poccia, N., Ricci, A., Fields, G., Burghammer, M., Aeppli, G., Bianconi, A.: Scale-free structural organization of oxygen interstitials in $\mathrm{La}_{2} \mathrm{CuO}_{4+y}$. Nature 466, 841-844 (2010).

[5] Poccia, N., Fratini, M., Ricci, A., Fields, G., Beard, L., Victorines-Orgeas, A., Bianconi, G., Aeppli, G., Bianconi, A.: Evolution and control of oxygen order in a cuprate superconductor. Nature Mater. 10, 733-736 (2011).

[6] Brookeman, J., Rigamonti, A.: Pretransitional clusters and heterophase fluctuations at first-order phase transitions in crystals. Phys. Rev. B 24, 4925-4930 (1981).

[7] Rigamonti, A.: NMR-NQR studies of structural phase transitions. Adv. Phys. 33, 115-191 (1984).

[8] Gordon, A., Genossar, J.: Precursor order clusters at ferroelectric phase transitions. Physica B 125, 53-62 (1984).

[9] Gordon, A.: Heterophase fluctuations in ferroelectrics. J. Phys. C 20, 111-114 (1987).

[10] Yukalov, V.I.: Heterophase fluctuations in ferroelectrics. Ferroelectrics, 82, 11-24 (1988).

[11] Gordon, A., Dorfman, S., Fuks, D.: Conspicuous domination of polarization relaxation in kinetics of first-order phase transitions in perovskites. Phys. Rev. B 54, 3055-3057 (1996).

[12] Gordon, A., Dorfman, S., Fuks, D.: Dynamic criterion in the kinetics of ferroelectric phase transitions. J. Phys. Condens. Matter 1996, 8, 385-390 (1996).

[13] Gordon, A., Dorfman, S., Fuks, D.: Competition between relaxation kinetics and heat transfer in the dynamics of phase transition fronts. J. Phys. France 7, 53-58 (1997).

[14] Yamada, Y., Iwase, T., Fujishiro, K., Uesu, Y., Yamashita, Y., Tomeno, I., Shimanuke, S.: Relaxor as heterophase fluctuation. Ferroelectrics 240, 363-370 (2000).

[15] Yukalov, V.I.: Phase transitions and heterophase fluctuations. Phys. Rep. 208, 395-492 (1991).

[16] Yukalov, V.I.: Mesoscopic phase fluctuations: general phenomenon in condensed matter. Int. J. Mod. Phys. B 17, 2333-2358 (2003).

[17] Gibbs, J.W.: Collected Works. Longmans, New York (1928), Vol. 1. 
[18] Gibbs, J.W.: Collected Works. Longmans, New York (1931), Vol. 2.

[19] Blinc, R., Zeks, B.: Soft Modes in Ferroelectrics and Antiferroelectrics. Elsevier, New York (1974).

[20] Yukalov, V.I., Yukalova, E.P.: Mesoscopic disorder in double-well optical lattices. Laser Phys. 21, 1448-1458 (2011).

[21] Benedek, G., Müller, K.A. (eds): Phase Separation in Cuprate Superconductors. World Scientific, Singapore (1992).

[22] Sigmund, E., Müller, K.A. (eds): Phase Separation in Cuprate Superconductors. Springer, Berlin (1994).

[23] Kivelson, S.A., Bindloss, I.P., Fradkin, E., Oganesyan, V., Tranquada, J.M., Kapitulnik, A., Howald, C.: How to detect fluctuating stripes in the high-temperature superconductors. Rev. Mod. Phys. 75, 1201-1241 (2003).

[24] Bednorz, G., Müller, K.A.: Possible high $T_{c}$ superconductivity in the Ba-La-Cu-O system. Z. Phys. B 64, 189-193 (1986).

[25] Shumovsky, A.S., Yukalov, V.I.: Microscopic model of a superconductor with normal-state nuclei. Dokl. Phys. 27, 709-711 (1982).

[26] Yukalov, V.I.: On the model of heterophase superconductor. JINR, Dubna (1985).

[27] Gorkov, L.P., Sokol, A.V.: Phase stratification of an electron liquid in the new superconductors. JETP Lett. 46, 420-423 (1987).

[28] Khait, Y.L.: A new kind of electron-lattice interaction and the kinetics of breaking high- $T_{c}$ and low- $T_{c}$ superconductivity via large thermal energy fluctuations of lattice particles. Z . Phys. B 71, 7-18 (1988).

[29] Yukalov, V.I.: Heterostructural fluctuations in superconductors. Int. J. Mod. Phys. B 6, 91-107 (1992).

[30] Coleman, A.J., Yukalova, E.P., Yukalov, V.I.: Superconductors with mesoscopic phase separation. Physica C 243, 76-92 (1995).

[31] Yukalov, V.I.: Mesoscopic phase separation in anisotropic superconductors. Phys. Rev. B 70, 224516 (2004).

[32] Ledbetter, H., Lei, M., Kim, S.: Elastic constants, Debye temperatures, and electronphonon parameters of superconducting cuprates and related oxides. Phase Trans. 23, 61-70 (1990).

[33] Ledbetter, H.: Dependence of $T_{c}$ on Debye temperature $\Theta_{D}$ for various cuprates. Physica C 235, 1325-1326 (1994).

[34] Zhao, G.M., Keller, H., Conder, K.: Unconventional isotope effects in the high-temperature cuprate superconductors. J. Phys. Condens. Matter 13, 569-587 (2001).

[35] Abd-Shukor, R.: Acoustic Debye temperature and the role of phonons in cuprates and related superconductors. Supercond. Sci. Technol. 15, 435-438 (2002). 